“ (C) 2017 IEEE. Personal use of this material is permitted. Permission from IEEE must be obtained for all other uses, in any current or future media, including

reprinting/republishing this material for advertising or promotional purposes, creating new collective works, for resale or redistribution to servers or lists, or reuse of any copyrighted component of this work in other works." 


\title{
Analog Antenna Array based Sensing in Perceptive Mobile Networks
}

\author{
Md. Lushanur Rahman, J. Andrew Zhang, Xiaojing Huang, and Y. Jay Guo*
}

\begin{abstract}
In this paper, we consider an alternative low-cost and flexible solution of using an analog antenna array for radio sensing at the base station receiver in the recently proposed perceptive mobile networks. We provide receiver beamforming design, and advanced compressive sensing (CS) signal processing techniques for sensing parameter estimation in a multiuser-MIMO (MU-MIMO) communications system. Simulation results are provided and validate the effectiveness of the proposed solution and sensing algorithms.
\end{abstract}

\section{INTRODUCTION}

The recently proposed perceptive mobile network [1] can provide integrated communication and radio sensing in one system. On a unified sensing platform, extraction of sensing parameters using both uplink and downlink signals is proposed. In order to achieve sensing using downlink signals, the transmitter and receiver at a remote radio unit (RRU) need to be able to operate simultaneously. A simple solution is to use separated antennas for transmitter and receiver. However, to obtain good estimation for sensing parameters, particularly, angle of arrivals of signals, a large number of receiving antennas, including extra antenna installation space, and RF chains are also required. In turns, these can significantly increase the cost of the receiver, although the transmitter can remain almost unchanged.

In this paper, we investigate the low-cost option of using an analog phased antenna array dedicated to the receiver for sensing and communications, and develop sensing parameter estimation algorithms, based on the same MU-MIMO model in [1].

We formulate theoretical framework for sensing problem in section 2, and we propose 1-D CS algorithm for parameter estimation in section 3 . Numerical results are provided and verify the effectiveness of the proposed scheme.

\section{PROBLEM FORMULATION}

We here focus on estimating spatial parameters including distance, direction, and speed of objects by extracting the composition of mobile signals.

\footnotetext{
*University of Technology Sydney, Global Big Data Technologies Center (GBDTC), Sydney, Australia, e-mail: MdLushanur.Rahman@student.uts.edu.au; A Andrew.Zhang; Xiaojing.Huang; Jay.Guo\}@uts.edu.au.
}

\subsection{System Model}

We consider a typical radio system, similar to [1], where $Q$ RRUs collaboratively facilitate MUMIMO service to $K$ users. Each RRU and each user have a linear antenna array of $M$ elements and $M_{T}$ elements, respectively. We used MIMOOFDMA type of modulation for both uplink and downlink. Let $N$ is the number of subcarriers and $B$ the total bandwidth. Then the subcarrier interval is $f_{0}=B / N$ and OFDM symbol period is $T_{s}=N / B+T_{p}$ where $T_{p}$ is the period of cyclic prefix. The array response vector of a size- $M$ array for narrowband signals is given by

$$
\mathbf{a}(M, \theta)=\left(1, e^{j \kappa \sin (\theta)}, \cdots, e^{j \kappa(M-1) \sin (\theta)}\right)^{T},
$$

where $\kappa=2 \pi d / \lambda, d$ is antenna interval in the array, $\lambda$ is the wavelength, and $\theta$ is either angle-of-depart (AoD) or angle-of-arrival (AoA).

For the $\ell$-th out of a total of $L$ multipath signals, let $\theta_{\ell}$ and $\phi_{\ell}$ be the AoD and AoA, respectively, and $b_{\ell}$ the amplitude, $\tau_{\ell}$ the propagation delay, and $f_{D, \ell}$ the associated Doppler frequency.

The basic task for sensing is to estimate these spatial parameters $\left\{\tau_{\ell}, f_{D, \ell}, \phi_{\ell}, \theta_{\ell}, b_{\ell}\right\}, \ell=1, \cdots, L$ from the received signals. We defined downlink and uplink sensing, as sensing performed at a RRU employing downlink transmitted signals from itself and other cooperative RRUs and uplink signals from users, respectively [1], [2]. Whereas they can have a similar mathematical formation [1]. In turns, intuitively, from downlink perspective, processed received signal model at a RRU at the $n$-th subcarrier and the $t$-th OFDM block can be represented as

$$
\begin{aligned}
\mathbf{Y}_{n, t}= & \sum_{q=1}^{Q} \sum_{\ell=1}^{L_{q}} b_{q, \ell} e^{-j 2 \pi n \tau_{q, \ell} f_{0}} e^{j 2 \pi t f_{D, q, \ell} T_{s}} \\
& \mathbf{a}\left(M, \phi_{q, \ell}\right) \mathbf{a}^{T}\left(M, \theta_{q, \ell}\right) \mathbf{x}_{q, n, t}+\mathbf{z}_{n, t}, \\
= & \mathbf{A}(M, \phi) \mathbf{C}_{n} \mathbf{D}_{t} \mathbf{U}^{T} \mathbf{x}_{n, t}+\mathbf{z}_{n, t}
\end{aligned}
$$

where variables with subscript $q$ are for the $q$-th $\mathrm{RRU}, \mathbf{x}_{q, n, t}$ are the transmitted signals at subcarrier $n$ from the $q$-th $\mathrm{RRU}, \mathbf{z}_{n, t}$ is the noise vector. Note, $\mathbf{A}(M, \boldsymbol{\phi})=\left(\mathbf{A}_{1}\left(M, \boldsymbol{\phi}_{1}\right), \cdots, \mathbf{A}_{Q}\left(M, \phi_{Q}\right)\right)$, $\mathbf{x}_{n, t}=\left(\mathbf{x}_{1, n, t}, \cdots, \mathbf{x}_{Q, n, t}\right)^{T}, \quad \mathbf{U}=$ $\operatorname{diag}\left\{\mathbf{A}_{1}\left(M, \boldsymbol{\theta}_{1}\right), \cdots, \mathbf{A}_{Q}\left(M, \boldsymbol{\theta}_{Q}\right)\right\}$, hence $\mathbf{U}$ is 
a $M Q \times L, L=\sum_{\ell=1}^{Q} L_{q}$, block diagonal matrix. The $\ell$-th column in $\mathbf{A}_{q}\left(M, \phi_{q}\right)$ (or $\left.\mathbf{A}_{q}\left(M, \boldsymbol{\theta}_{q}\right)\right)$ is $\mathbf{a}\left(M, \phi_{q, \ell}\right)\left(\right.$ or $\left.\mathbf{a}\left(M, \theta_{q, \ell}\right)\right), \mathbf{D}_{t}$ and $\mathbf{C}_{n}$ are diagonal matrices with the $\sum_{q^{\prime}=1}^{q-1} L_{q^{\prime}}+\ell$-th diagonal element being $b_{\ell} e^{j 2 \pi t f_{D, q, \ell} T_{s}}$ and $e^{-j 2 \pi n \tau_{q, \ell} f_{0}}$, respectively.

\subsection{Generalized Delay-Quantized On-grid Formulation}

We assume $N \gg L$ to reduce the quantization error of $\tau_{\ell}$ and the delay estimation can be well approximated as an on-grid estimation problem. Let $e^{-j 2 \pi n \tau_{\ell^{\prime}} f_{0}}$ be quantized to $e^{-j 2 \pi n \ell /\left(N^{\prime}\right)}$, where $N^{\prime}$ can equal to or be multiple times of $N$, implies the minimal resolvable delay as $1 / N^{\prime}$.

Let $K$ and $M_{T}$ denote the total number of users/RRUs and number of antennas in each user/RRU, respectively, for either uplink or downlink sensing. We now convert the multipath signal models of both uplink and downlink to a generalized on-grid (delay only) sparse model, by taking $N_{p} \gg L, N_{p} \leq N^{\prime}$ multipath signals where only $L$ signals are non-zeros. Referring to (3), after applying receiver beamforming with $\mathbf{Y}_{n, t}$, the delay-ongrid received signal model can be represented as

$$
y_{n, t}=\mathbf{g}_{t}^{T} \mathbf{A}(M, \phi) \mathbf{C}_{n} \mathbf{D}_{t} \mathbf{P} \mathbf{U}^{T} \mathbf{x}_{n, t}+\mathbf{z}_{n, t},
$$

where $\mathbf{g}_{t}$ is the $M \times 1$ beamforming vector applied to the $t$-th OFDM block, $\mathbf{C}_{n}$ redefined as $\mathbf{C}_{n}=\operatorname{diag}\left\{1, e^{-j 2 \pi n / N^{\prime}}, \cdots, e^{-j 2 \pi n\left(N_{p}-1\right) / N^{\prime}}\right\}, \mathbf{P}$ is an $N_{p} \times L$ rectangular permutation matrix that maps the signals from a user/RRU to its multipath signal, $\mathbf{U}$ is an $M_{T} K \times L$ matrix while $\mathbf{x}_{n, t}$ is the $M_{T} K \times 1$ symbol vector; the other symbols have similar expressions with those in (3), with the columns in $\mathbf{A}(M, \phi)$ of size $M \times N_{p}$ and the diagonal elements in $\mathbf{D}_{t}$ of size $N_{p} \times N_{p}$ are reordered as well. Here, we allow repeated delay values in $\mathbf{C}_{n}$ to account for multipath signals with the same quantized delay but different AoAs and/or AoDs.

The design of beamforming vectors $\mathbf{g}_{t}$ depends on factors such as energy collection and AoA estimation algorithms. Without any prior knowledge about the AoA, a simple and efficient way is to take it from equally-spaced columns of an $M \times M$ DFT matrix

$$
\mathbf{F}=\left\{e^{-j 2 \pi m t^{\prime} / M}\right\}, m, t^{\prime}=0, \cdots, M-1 .
$$

Assume that channel parameters are fixed during $T=M / c_{1}$ OFDM blocks where $c_{1}$ is an integer. Then at the phased array we repeatedly apply $T$ $M \times 1$ beamforming vectors

$\mathbf{g}_{t}=\left\{e^{-j 2 \pi m\left(t_{0}+\bmod (t, T) c_{1}\right) / M}\right\}, m=0, \cdots, M-1$,

where $t_{0}$ is an initial offset value, and $\bmod (\cdot, \cdot)$ is the modulus operator.

\section{ESTIMATION OF SPATIAL PARAME- TERS}

We now demonstrate a scheme based on 1-D CS for estimating the spatial parameters. For uplink sensing, symbols $\mathbf{x}_{n}$ s can be achieved by demodulating them as sensing can tolerate more delay than communication. On the contrary, for downlink sensing, they are centrally known. Note, the range of subcarriers in downlink and uplink sensing could be different. RRUs can obtain signals at more subcarriers in downlink sensing than uplink, as the total subcarriers may shared by different group of users.

\subsection{Single Multipath for Each Delay}

Rewrite (4) as

$$
y_{n, t}^{T}=\mathbf{x}_{n, t}^{T}\left(\mathbf{c}_{n}^{T} \otimes \mathbf{I}_{M_{T} K}\right) \mathbf{V}_{t} \mathbf{A}^{T}(M, \phi) \mathbf{g}_{t},
$$

where $\otimes$ denotes the Kronecker product, $\mathbf{c}_{n}=$ $\left(1, e^{-j 2 \pi n / N^{\prime}}, \cdots, e^{-j 2 \pi\left(N_{p}-1\right) / N^{\prime}}\right)^{T}, \quad \mathbf{I}_{M_{T} K}$ is an $M_{T} K \times M_{T} K$ identity matrix, and $\mathbf{V}$ is a $M_{T} K N_{p} \times N_{p}$ block diagonal matrix with $\mathbf{p}_{\ell}$ being the $\ell$-th column of $\mathbf{P}^{T}$. Note,

$$
\mathbf{V}_{t}=\operatorname{diag}\left\{b_{\ell} e^{j 2 \pi t f_{D, \ell} T_{s}} \mathbf{U} \mathbf{p}_{\ell}\right\}_{\ell=1, \cdots, N_{p}}
$$

We have now separated signals $\mathbf{x}_{n, t}^{T}\left(\mathbf{c}_{n}^{T} \otimes \mathbf{I}_{M_{T} K}\right)$ that are known and dependent on $n$ from others. Let $\mathcal{S}_{s}$ denote the set of available subcarriers for sensing and let $N_{s}$ denote its size. Stacking $N_{s}$ $y_{n, t}^{T}, n \in \mathcal{S}_{s}$ to a vector generates

$$
\mathbf{y}_{t}=\mathbf{W}_{t} \mathbf{V}_{t} \mathbf{A}^{T}(M, \phi) \mathbf{g}_{t},
$$

where $\mathbf{W}_{t}$ is an $N_{s} \times M_{T} K N_{p}$ matrix with its $n$-th row being $\mathbf{x}_{n, t}^{T}\left(\mathbf{c}_{n}^{T} \otimes \mathbf{I}_{M_{T} K}\right)$.

Inspecting (8), we can see that the estimation problem can be formulated as a block sparse problem [3] with $N_{s} \times 1$ observations $\mathbf{y}_{t}$, sensing matrix $\mathbf{W}_{t}$, and block sparse signals $\mathbf{V}_{t} \mathbf{A}^{T}(M, \phi) \mathbf{g}_{t}$ of $L$ sparsity. Let $\mathbf{V}_{t}=\left(\mathbf{V}_{1}^{T}, \mathbf{V}_{2}^{T}, \cdots, \mathbf{V}_{N_{p}}^{T}\right)^{T}$ where $\mathbf{V}_{\ell}$ denotes the $M_{T} K \times N_{p}$ block signals, and $L$ out of $N_{p} \mathbf{V}_{\ell}$ s have non-zero elements. A block sparse CS algorithm can solve (8) and generate estimates for $\mathbf{V}_{\ell} A^{T}(M, \phi) \mathbf{g}_{t}, \ell=1, \cdots, N_{p}$.

We first consider noiseless cases. Once the $L$ nonzero blocks $\mathbf{V}_{\ell} \mathbf{A}^{T}(M, \phi) \mathbf{g}_{t}$ are determined, we can then get the $L$ delay estimates according to the indexes of the blocks.

In (7), only the $\ell$-th column in $\mathbf{V}_{\ell}$ has non-zero elements $b_{\ell} e^{j 2 \pi t f_{D, \ell} T_{s}} \mathbf{U} \mathbf{p}_{\ell}$ if $b_{\ell} \neq 0$. Therefore,

$$
\mathbf{V}_{\ell} \mathbf{A}^{T}(M, \phi) \mathbf{g}_{t}=b_{\ell} e^{j 2 \pi t f_{D, \ell} T_{s}} \mathbf{U p}_{\ell} \mathbf{a}^{T}\left(M, \phi_{\ell}\right) \mathbf{g}_{t} .
$$

Since $\mathbf{p}_{\ell}$ only has a single non-zero element $1, \mathbf{U} \mathbf{p}_{\ell}$ will generate a column vector corresponding to one 
column in $\mathbf{U}$. As $\mathbf{U}$ is a block diagonal matrix, only 1 out of $K M_{T} \times 1$ vectors in each column is non-zero.

Now represent $\mathbf{V}_{\ell} \mathbf{A}^{T}(M, \phi) \mathbf{g}_{t}$ as $K M_{T} \times 1$ subvectors $\left(\mathbf{b}_{\ell, 1, t}^{T}, \cdots, \mathbf{b}_{\ell, K, t}^{T}\right)^{T}$. If $\mathbf{b}_{\ell, k, t} \neq 0$, then this multipath is from the $k$-th RRU (user). Hence,

$$
\mathbf{b}_{\ell, k, t}=b_{\ell} e^{j 2 \pi t f_{D, \ell} T_{s}} \mathbf{a}\left(M_{T}, \theta_{k, \ell}\right) \mathbf{a}^{T}\left(M, \phi_{k, \ell}\right) \mathbf{g}_{t} .
$$

From $\mathbf{b}_{\ell, k, t}$, calculating the cross-correlation between rows, we can obtain AoD estimates. The mean energy of $\mathbf{b}_{\ell, k, t}$ can also be estimated directly via the cross-correlation output.

When the channel parameters remain fixed over at least $2 T$ OFDM blocks, we can calculate the cross correlation and use the following equation to estimate Doppler shift:

$$
f_{D, \ell}=\angle\left(\sum_{t=t_{1}}^{t_{1}+T-1}\left(\mathbf{b}_{\ell, k, t}^{H} \mathbf{b}_{\ell, k, t+T}\right)\right) /\left(2 \pi T_{s} T\right),
$$

where we have exploited the fact that $T s T f_{D, \ell}$ is practically much smaller than 1 and hence there is no phase ambiguity here.

For the estimation of AoA, we can exploit $\mathbf{b}_{\ell, k, t} \mathrm{~s}$ across $T$ OFDM symbols. We can either ignore the phase variation caused by Doppler shift and process the signals per each transmitter antenna, or apply the estimated AoD and Doppler shift $f_{D, \ell}$ to (10) to improve signal energy. Consider the latter as an example. For $M-T>1, \mathbf{b}_{\ell, k, t}$ can be leftmultiplied with $e^{j 2 \pi t f_{D, \ell} T_{s}} \mathbf{a}^{H}\left(M_{T}, \theta_{k, \ell}\right)$ to remove Doppler shift and AoD terms, and to combine signals from different transmit antennas. Stacking the resulted signals over the first $T$ blocks into a $T \times 1$ vector, we get

$$
\mathbf{q}_{\ell, k}=M_{T} b_{\ell} \mathbf{G}^{T} \mathbf{a}\left(M, \phi_{k, \ell}\right),
$$

where $\mathbf{G}$ is an $M \times T$ matrix with the $(\bmod (t, T)+$ 1 )-th column being $\mathbf{g}_{t}$. The signal $\mathbf{q}_{\ell, k}$ can be further averaged over the first and second $T$ blocks.

When $T<M$, the pseudo-inverse of $\mathbf{G}^{T}$ does not exist, and hence $\mathbf{a}\left(M, \phi_{k, \ell}\right)$ cannot be obtained directly. We propose to use the following approach to estimate $\phi_{k, \ell}$ instead.

Note that $\mathbf{g}_{t}^{T} \mathbf{a}\left(M, \phi_{k, \ell}\right)$ can be computed as

$$
\begin{aligned}
& \mathbf{g}_{t}^{T} \mathbf{a}\left(M, \phi_{k, \ell}\right) \\
= & \sum_{m=0}^{M-1} e^{j 2 \pi m\left(\alpha-\left(t_{0}+\bmod (t, T) c_{1}\right) / M\right)} \\
= & \sum_{t^{\prime}=}^{T-1} \underbrace{e^{j 2 \pi t^{\prime}\left(\alpha-t_{0} / M\right)} \frac{1-e^{j 2 \pi M\left(\alpha-t_{0} / M\right)}}{1-e^{j 2 \pi T\left(\alpha-t_{0} / M\right)}}}_{\rho_{t^{\prime}}} \cdot R
\end{aligned}
$$

where $\alpha \triangleq d \sin \left(\phi_{k, \ell}\right) / \lambda$ and $R=e^{-j 2 \pi t^{\prime} \bmod (t, T) / T}$. Note that this is a T-point DFT of $\rho_{t^{\prime}}$, and in $\rho_{t^{\prime}}$, only $e^{j 2 \pi t^{\prime}\left(\alpha-t_{0} / M\right)}$ depends on $t^{\prime}$.

Hence, we can apply inverse DFT to $\mathbf{q}_{\ell, k}$ in (12) and get $\left\{\rho_{t^{\prime}}\right\}, t^{\prime}=1, \cdots, T$, and then get the AoA estimation through computing

$$
\sin \left(\phi_{k, \ell}\right)=\frac{\lambda}{2 \pi d} \angle\left(e^{j 2 \pi t_{0} / M} \sum_{t^{\prime}=1}^{T-1} \rho_{t^{\prime}+1} \rho_{t^{\prime}}^{*}\right),
$$

where $(\cdot)^{*}$ denotes the conjugate operator. Note that $M_{t} b_{\ell}$ in $\mathbf{q}_{\ell, k}$ is absorbed to $\rho_{t^{\prime}}$ during this calculation and has no impact on the result through the angle operation.

Once all other parameters are estimated in (12), $b_{\ell}$ can then be obtained too.

In noisy cases, we can sort the blocks $\mathbf{V}_{\ell} \mathbf{A}^{T}(M, \phi) \mathbf{g}_{t}, \ell=1, \cdots, N_{p}$ according to the energy of the block signals and use a threshold to pick up blocks with larger energy and corresponding to efficient multipath signals. This threshold may be determined by measuring the mean power across $T$ OFDM blocks when beamforming completes one cycle of scanning.

\subsection{Multiple Multipath Signals with the Same Delay}

Similar to those shown for MIMO systems in [1], if $\ell \in \mathcal{S}_{d}$ multipath signals have the same delays but different AoAs or AoDs, we will get

$$
\begin{aligned}
& \mathbf{V}_{\ell} \mathbf{A}^{T}(M, \boldsymbol{\phi}) \mathbf{g}_{t} \\
= & \sum_{\ell \in \mathcal{S}_{d}} b_{\ell} e^{-j 2 \pi t f_{D, \ell} T_{s}} \mathbf{U} \mathbf{p}_{\ell} \mathbf{a}^{T}\left(M, \phi_{\ell}\right) \mathbf{g}_{t}
\end{aligned}
$$

If these multipath signals are from different RRUs (users), multiple $\mathbf{b}_{\ell, k, t} \mathrm{~s}$ will be non-zero. Hence, these multipath signals can be estimated straightforwardly, by applying the results from (10) to the end of Subsection 3.1 to each user separately. To determine which users' multipath signals are located in one delay bin, both the largest power of blocks in one delay bin and average powers across the delays bins for a particular user may be exploited. Detailed design remains as an open question. In simulations we use a threshold corresponding to $50 \%$ of the mean signal energy in each delay bin identified containing efficient multipath signals.

A very challenging scenario is when $L_{\ell, k}>1 \mathrm{mul}$ tipath signals are from the same RRU (user). So,

$$
\begin{aligned}
\mathbf{b}_{\ell, k, t} & =\sum_{\ell \in \mathcal{S}_{d}} b_{\ell} e^{-j 2 \pi t f_{D, \ell} T_{s}} \mathbf{a}\left(M_{T}, \theta_{k, \ell}\right) \mathbf{a}^{T}\left(M, \phi_{k, \ell}\right) \mathbf{g}_{t} \\
& =\mathbf{A}_{T} \mathbf{D} \mathbf{A}_{R}^{T} \mathbf{g}_{t},
\end{aligned}
$$


where $\mathbf{A}_{T}$ is an $M_{T} \times L_{\ell, k}$ matrix with columns $\mathbf{a}\left(M_{T}, \theta_{k, \ell}\right), \mathbf{A}_{R}$ is an $M \times L_{\ell, k}$ matrix with columns $\mathbf{a}\left(M, \phi_{k, \ell}\right), \mathbf{D}$ is a diagonal matrix with diagonal elements $b_{\ell} e^{-j 2 \pi t f_{D, \ell} T_{s}}$, all with $\ell \in \mathcal{S}_{d}$.

When the numbers of antennas $M_{T}$ and $M$ are large, it is possible to apply CS technique to estimate both AoA and AoD, by working over $T$ OFDM blocks and ignoring the change of Doppler shift between them. When $M_{T}$ and $M$ are not very large but are larger than two times of the multipath number, the AoD can be estimated using spectrum analysis techniques such as ESPRIT or MUSIC using individual $\mathbf{b}_{\ell, k, t}$ or across $T \mathbf{b}_{\ell, k, t} \mathrm{~s}$. However, there seems no efficient spectrum analysis techniques or other techniques to accurately estimating AoA for the signals in (16), when $T<M$. A qualitative approach is to determine a coarse AoA according to the signal energy of $\mathbf{b}_{\ell, k, t}$ and the beamforming scanning direction.

When $T \geq M$ beamforming scanning over a stable channel period is possible (phase variation due to Doppler is ignored), we can stack $T \mathbf{b}_{\ell, k, t}$ s into an matrix and remove $\mathbf{g}_{t} \mathrm{~s}$ impact via right multiplying the matrix with the (pseudo) inverse of the $M \times T$ matrix $\left\{\mathbf{g}_{t}\right\}$. Then, we get a standard expression for which conventional spectrum analysis techniques or 2-D DFT analysis can be applied.

\section{SIMULATION RESULTS}

We present simulation results using the block Bayesian Sparse Learning algorithm [3] to validate the effectiveness of our parameter estimation scheme. We consider a system with 4 RRUs, each with a 8-element antenna array, providing connection to 4 users, each with 2 antennas. The carrier frequency is $2.35 \mathrm{GHz}, N=256, d=\lambda / 2$, and $B=100 \mathrm{MHz}$. No radar cross-section information is used. The total thermal noise in the receiver is $-97 \mathrm{dBm}$, the transmission power is $25 \mathrm{dBm}$ and the used pathloss model is same to [2]. Multipath signals for each RRU/MS are formed randomly in a cluster, mimicking reflected/scattered signals from objects. Each cluster is generated following uniform distributions of $[3,5]$ for the total multipath number and AoAs, AoDs and Doppler frequencies are randomly generated within a given range. Delays, from the same RRU/MS are different while same between RRUs/MSs. On-grid delay interval of $10 \mathrm{~ns}$ refers to a distance resolution of $3 \mathrm{~m}$.

Figure 1 displays the simulation results for parameter estimation with $T=4$. For downlink sensing, in (a), (b), all subcarriers are used. It shows that AOD and AOA estimates are accurately placed with the actual ones. For uplink sensing, in (c), (d), 64 randomly selected subcarriers are used by 4
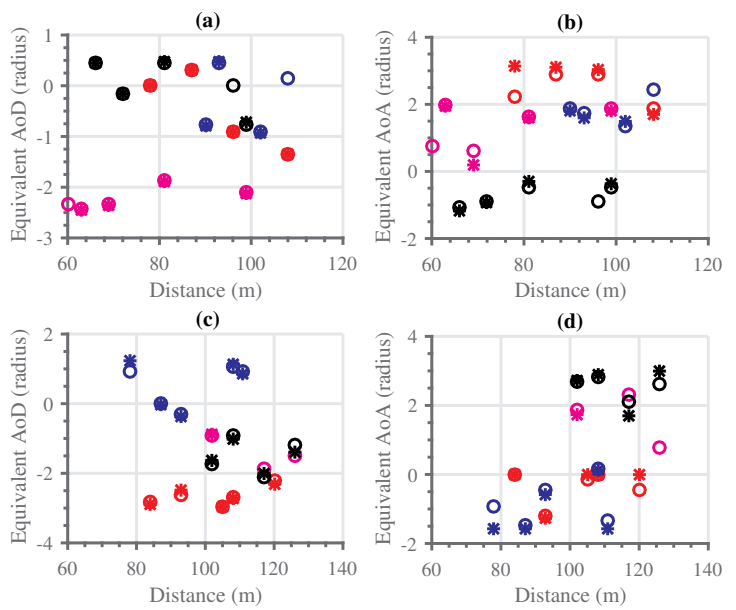

Figure 1: Parameter estimation for downlink sensing shows in (a), (b), while (c) and (d) refers to uplink sensing. Every star/circle refers parameters for one multipath; Stars and circles are for estimated and actual ones, respectively. Different colors represent multipath from different RRUs in (a), (b) and users in (c), (d).

users simultaneously. Estimates for this are with a few mismatched cases, especially the AOA (Figure 1(d)) with three circles. It reveals that, downlink sensing exploits centrally available signals at more subcarriers at static RRUs with larger arrays. However, in uplink, diverse resource allocation with random multiuser access draws an overall impact on estimation accuracy.

\section{CONCLUSIONS}

This paper presents a basic spatial parameter estimation method based on analog antenna array for a sensing system unified with communication. A compressive scheme is developed for estimation, and its effectiveness is validated by simulation results. Our work here provides a step forward in demonstrating the feasibility of analog array in receiver cost minimization for perceptive network.

\section{References}

[1] J. A. Zhang, A. Cantoni, X. Huang, Y. J. Guo, and R. W. H. Jr., "Framework for an Innovative Perceptive Mobile Network Using Joint Communication and Sensing," in VTC Spring $201 \%$.

[2] J. A. Zhang, A. Cantoni, X. Huang, Y. J. Guo, and R. W. H. Jr., "Joint Communications and Sensing Using Two Steerable Analog Antenna Arrays," in VTC Spring 2017, June 2017.

[3] S. Ji, Y. Xue, and L. Carin, "Bayesian compressive sensing," IEEE Trans. Signal Process, vol. 56, no. 6, pp. 2346-2356, June 2008. 